\title{
Von Hippel-Lindau mutations disrupt vascular patterning and maturation via Notch
}

\author{
Alexandra Arreola, ${ }^{1}$ Laura Beth Payne, ${ }^{2}$ Morgan H. Julian, ${ }^{2,3}$ Aguirre A. de Cubas, ${ }^{4}$ \\ Anthony B. Daniels, ${ }^{5,6,7,8}$ Sarah Taylor, ${ }^{2}$ Huaning Zhao, ${ }^{2,9}$ Jordan Darden, ${ }^{2,10}$ Victoria L. Bautch, ${ }^{1,11,12}$ \\ W. Kimryn Rathmell, ${ }^{4,6,8}$ and John C. Chappell2,3,9,10 \\ 'Lineberger Comprehensive Cancer Center, University of North Carolina at Chapel Hill (UNC-CH), Chapel Hill, North Carolina, \\ USA. ${ }^{2}$ Center for Heart and Regenerative Medicine and ${ }^{3}$ Department of Basic Science Education, Virginia Tech Carilion \\ School of Medicine and Research Institute, Roanoke, Virginia, USA. ${ }^{4}$ Department of Medicine, Division of Hematology \\ and Oncology, ${ }^{5}$ Department of Ophthalmology and Visual Sciences, ${ }^{6}$ Department of Biochemistry, ${ }^{7}$ Department of \\ Radiation Oncology, and ${ }^{8}$ Vanderbilt-Ingram Cancer Center, Vanderbilt University Medical Center, Nashville, Tennessee, \\ USA. ${ }^{9}$ Department of Biomedical Engineering and Mechanics, ${ }^{10} \mathrm{Graduate}$ Program in Translational Biology, Medicine, \\ and Health, Virginia Polytechnic Institute and State University, Blacksburg, Virginia, USA. "Department of Biology and \\ ${ }^{12}$ McAllister Heart Institute, UNC-CH, Chapel Hill, North Carolina, USA.
}

Von Hippel-Lindau (VHL) gene mutations induce neural tissue hemangioblastomas, as well as highly vascularized clear cell renal cell carcinomas (ccRCCs). Pathological vessel remodeling arises from misregulation of HIFs and VEGF, among other genes. Variation in disease penetrance has long been recognized in relation to genotype. We show Vhl mutations also disrupt Notch signaling, causing mutation-specific vascular abnormalities, e.g., type 1 (null) vs. type 2B (murine G518A representing human $\mathrm{R} 167 \mathrm{Q}$ ). In conditional mutation retina vasculature, Vhl-null mutation (i.e., $\left.U B C^{C r e E R /+} V h I^{f / f f}\right)$ had little effect on initial vessel branching, but it severely reduced arterial and venous branching at later stages. Interestingly, this mutation accelerated arterial maturation, as observed in retina vessel morphology and aberrant $\alpha$-smooth muscle actin localization, particularly in vascular pericytes. RNA sequencing analysis identified gene expression changes within several key pathways, including Notch and smooth muscle cell contractility. Notch inhibition failed to reverse later-stage branching defects but rescued the accelerated arterialization. Retinal vessels harboring the type $2 \mathrm{~B} \mathrm{Vhl}$ mutation (i.e., UBC ${ }^{\mathrm{CreER} /+} \mathrm{Vh} \mathrm{h}^{\mathrm{f} / 2 \mathrm{2B}}$ ) displayed stage-specific changes in vessel branching and an advanced progression toward an arterial phenotype. Disrupting Notch signaling in type 2B mutants increased both artery and vein branching and restored arterial maturation toward nonmutant levels. By revealing differential effects of the null and type $2 \mathrm{~B} \mathrm{Vh/} \mathrm{mutations} \mathrm{on} \mathrm{vessel}$ branching and maturation, these data may provide insight into the variability of VHL-associated vascular changes - particularly the heterogeneity and aggressiveness in ccRCC vessel growth - and also suggest Notch pathway targets for treating VHL syndrome.

Conflict of interest: The authors have declared that no conflict of interest exists.

Submitted: December 9, 2016 Accepted: January 18, 2018 Published: February 22, 2018

Reference information: JCI Insight. 2018;3(4):e92193. https:// doi.org/10.1172/jci.insight.92193.

\section{Introduction}

Von Hippel-Lindau (VHL) syndrome is an autosomal dominant inherited predisposition to the onset of cancer in neurological tissues, as well as in the kidney, adrenal gland, pancreas, and liver (1). Genetic lesions that compromise the normal VHL protein ( $p$ VHL) function can give rise to different types of carcinomas, such as clear cell renal cell carcinoma (ccRCC) (2). In addition to elevated risk for ccRCC, $60 \%$ $80 \%$ of patients with VHL syndrome, including those with type $2 \mathrm{~B}$ missense VHL mutations, will develop hemangioblastomas (3-5). These benign neoplasms are composed of proliferating endothelial and stromal cells that arise in the brain and brain stem, as well as the spinal cord and retina, and often result in catastrophic effects for patients due to their site of presentation (6). Among other functions, the ubiquitin E3 ligase substrate recognition activity of $\mathrm{pVHL}$ provides essential modulation of hypoxia-inducible factors (HIFs), which orchestrate transcription of downstream gene targets in proportion to tissue oxygen levels. The VHL type 2B mutation R167Q has been described as a loss of pVHL interaction with Elongin C but 
retention of partial regulation of HIF- $1 \alpha$ and HIF-2 $\alpha$ levels, which may help explain the observed clinical spectrum (7). Numerous HIF-1 $\alpha$ targets are key regulators of blood vessel growth and remodeling, the most potent of which is VEGF-A (8).

Endothelial cells respond to VEGF-A through the receptor tyrosine kinase Flk-1/VEGF Receptor-2 (VEGFR-2), which promotes endothelial cell proliferation and sprouting migration (9). The Notch pathway intersects with VEGF-A signaling to coordinate endothelial cell behaviors such that endothelial "tip" cells emigrate outward from parent blood vessels, while "stalk" cells largely divide to promote vessel elongation $(10,11)$. Genetic loss of delta-like 4 (D114) or pharmacological blockade of Notch1 receptor signaling, such as through $\mathrm{N}$-[N-(3,5-Difluorophenacetyl)-L-alanyl]-S-phenylglycine t-butyl ester (DAPT) treatment, leads to a hypersprouting phenotype and the formation of excessive vessel branches $(10,12)$, though this nascent vasculature is not always functional $(13,14)$. Endothelial cells also express the Notch ligand Jagged1 (Jag1), an antagonist of D114 that competes for binding of Notch1 and thus modulates tip cell formation and vascular network complexity (15). Notch signaling also specifies arterial-venous identity of endothelial cells (16), providing key signals for vessel maturation and mural cell development (17-19). For instance, endothelial Jag1 induces mural cell expression of vascular smooth muscle cell (SMC) proteins such as $\alpha$-smooth muscle actin ( $\alpha$ SMA) via binding of the Notch3 receptor $(20,21)$. These, and numerous other studies, therefore support a model in which elevated Notch signaling restricts overall branching complexity of a developing network while promoting vessel maturation and arterialization (22); thus, Notch blockade can increase vessel branching and limit processes involved in maturation, such as the acquisition of functional vascular SMCs $(19,21)$. The effect of Notch blockade in the setting of variable levels of pathogenic pathway activation, however, is unknown.

Aberrant Notch signaling has been implicated in pathological conditions associated with VHL syndrome, most notably in the onset and progression of $\operatorname{ccRCC}(23,24)$. While $V H L$ mutations are known to disrupt the HIF/VEGF-A signaling axis and lead to abnormal vascular remodeling (25), the intersection with the Notch pathway, particularly in the vascular compartment, remains to be fully elucidated in the VHL mutation background. Recent studies suggest solid tumors frequently acquire a resistance to antiangiogenic, and specifically anti-VEGF, therapies $(26,27)$; therefore, a more complete understanding of Notch signaling in the setting of $V H L$ loss or mutation is critical for developing alternative therapeutic targets within the Notch pathway for treating ccRCC and VHL-related conditions such as hemangioblastoma.

In the current study, we explored blood vessel development in the context of conditional biallelic $\mathrm{Vhl}$ loss (i.e., $U B C^{\mathrm{CreER} /+} V h h^{f / f f}$ ), as well as in the setting of a specialized model of type 2B Vhl-knock-in mutation, rendered monoallelic by conditional deletion of a WT copy of $V h l$ (i.e., $U B C^{C r e E R /+} V h t^{7 / 2 B}$ ), mimicking the loss of heterozygosity that takes place in human VHL disease (referred to as VHL type 2B mutant). Embryonic stem cell-derived (ES cell-derived) blood vessels harboring the type 2B G518A missense mutation in $\mathrm{Vhl}$ (equivalent to the arginine 167 to alutamine [R167Q] in humans) displayed a more severe vascular dysmorphogenesis than the $\mathrm{Vht}^{-1-}$ vasculature. Endothelial cells isolated from both genotypic backgrounds, however, exhibited transcriptional alterations in VEGF-A and Notch pathway genes. In vivo analysis of the early stages of mouse retinal vessel development P5 revealed branching morphology defects in the setting of a $V h l$ type $2 \mathrm{~B}$ mutation (i.e., $U B C^{+/+} V h l^{7 / 2 B}$ and $U B C^{C r e E R /+} V h l^{7 / 2 B}$ ) but only subtle changes in vessels of conditionally deleted $V h l$-null mice (i.e., $U B C^{\mathrm{CreER} /+} V h \boldsymbol{l}^{\mathrm{tl} / \mathrm{f}}$ ). At slightly later stages of retinal development (P7), we found that genetic loss of $\operatorname{Vhl}$ (i.e., $U B C^{\mathrm{CreER} /+} V h h^{f / f f}$ ) caused an aberrant increase in arterial maturation (e.g., premature elevation of pericyte $\alpha$ SMA expression) at the expense of expanding capillary density. $V h l$ type 2B mutation carriers induced to lose the WT copy of $V h l$ (i.e., $U B C^{C r e E R /+} V h t^{f / 2 B}$ ), however, exhibited this accelerated arterial formation without a concomitant decrease in retinal microvessels. Pharmacological inhibition of Notch signaling rescued the aberrant arterial maturation phenotype while causing significant increases in vessel branching. Applying RNA Sequencing (RNA-Seq) analysis to these $V H L$ mutants, we found distinct changes in gene expression patterns of key signaling networks, including the Notch, HIF, SMC contraction, and FoxO/TGF $\beta$ pathways. Toward the completion of retinal development at $\mathrm{P} 21$, conditionally $V h l$-null and type $2 \mathrm{~B}$ mutant (i.e., $U B C^{\mathrm{CreER} /+} V h f^{f / f l}$ and $U B C^{\mathrm{CreER} /+} V h t^{f / 2 B}$, respectively) vasculature displayed severe morphological defects, reflecting misregulation within the Notch pathway that integrates with and compounds defects in these other critical signaling cascades. Taken together, these results demonstrate that the type $2 \mathrm{~B} V H L$ mutation may influence vascular changes and hemangioblastoma formation by accelerating the maturation of larger-caliber arteries in addition to maintaining and expanding capillary density, which could, in turn, worsen pathological progression via blood perfusion defects. 


\section{Results}

Type $2 B$ Vhl mutation disrupts VEGF and Notch signaling and causes blood vessel-branching defects. The VHL complex provides essential regulation of HIFs, which in turn modulate expression of a number of downstream proangiogenic target genes, including VEGF-A. Because Notch signaling intersects with the VEGF-A pathway $(10,11)$, we hypothesized that complete genetic loss of $V h l\left(V h l^{--}\right)$and the type 2B Vhl mutation cause vessel overgrowth through downstream disruptions in both the VEGF-A and Notch pathways. Endothelial cells that arise during mouse ES cell differentiation undergo angiogenic sprouting and form lumenized blood vessels, akin to vascular development in vivo $(28,29)$. We analyzed WT ES cell-derived vascular networks for their overall branching complexity and compared them with vessels formed in the $\mathrm{Vhl}$-null $\left(\mathrm{Vhl}^{--}\right)$and -type 2B mutant backgrounds (Supplemental Figure 1; supplemental material available online with this article; https://doi.org/10.1172/jci.insight.92193DS1). Interestingly, $\mathrm{Vht}^{\mathrm{t}^{--}}$vessels did not exhibit any significant changes in overall vessel branching. In contrast, type $2 \mathrm{~B}$ mutant (i.e., $V h h^{2 B / 2 B}$ ) vasculature showed a significant increase in branching complexity, suggesting that the type $2 \mathrm{~B}$ mutation has distinct effects on the molecular mechanisms governing blood vessel branching.

To further test our hypothesis regarding VEGF-A and Notch pathway disruptions downstream of genetic $V h l$ mutations, we analyzed the transcriptional profile of WT, $V h t^{-1}$, and $V h t^{2 B / 2 B}$ endothelial cells by assessing candidate targets within these pathways. We enzymatically dissociated ES cell cultures from each genetic background and used magnetic bead-assisted cell sorting (i.e., via platelet-endothelial cell adhesion molecule-1 [PECAM-1]/CD31 labeling) to enrich for endothelial cells. We assessed mRNA levels by quantitative PCR (qPCR) specifically evaluating VEGF-A and VEGFR2/Flk-1 levels, as well as the Notch pathway components D114, Jag1, Notch3, and Hey2 (Supplemental Figure 1). Although VEGFR2/ Flk-1 mRNA levels were significantly higher in both $V h l$ mutant backgrounds, only the type 2B Vhl mutant endothelial cells harbored a significant increase in VEGF-A transcript levels. Interestingly, D114 mRNA levels were significantly lower in $\mathrm{Vht}^{-1-}$ endothelial cells, as compared with WT, while these transcripts were significantly elevated in the type $2 \mathrm{~B}$ mutants. Endothelial Notch3 expression was significantly higher in both backgrounds, and Jag1 expression was raised in both but showed a significant increase only in the $V h t^{-1}$ context. The Notch pathway transcription factor Hey2 was significantly elevated in endothelial cells only expressing type 2B $V h l$ (i.e., $V h l^{2 B / 2 B}$ ); however, it was unchanged with full genetic loss of $V h l$. Taken together, these observations suggest that the $V h l^{-1}$ and type 2B $V h l$ mutations elicit specific effects on endothelial cells, which is reflected in expression of VEGF-A and Notch pathway components, and these distinct signaling environments yield vascular networks with overall unique morphologies.

Conditional Vhl mutations induce abnormal blood vessel development in early-stage postnatal mouse retinas. In observing differential effects of $\mathrm{Vht}^{-1-}$ and $\mathrm{Vhl}^{2 B / 2 B}$ on vascular development, we hypothesized that in vivo blood vessel formation would also exhibit distinct abnormalities due to disruption of the VEGF-A and Notch signaling pathways. To test this hypothesis, we assessed blood vessel formation in the developing retinas of P5 mice harboring (i) tamoxifen-inducible Cre-recombinase activity (i.e., $U B C^{C r e E R /+}$ ) and (ii) loxP sites for creating mutated $V h l$ alleles via targeted recombination ( $V h t^{f / f l}$ and $V h l^{f / 2 B}$; Supplemental Figure 2). After tamoxifen administration from P1-P3, retinas were collected at P5 and immunostained to visualize the developing vascular networks using confocal microscopy. Arterial and venous vasculature in the $V h l$ type $2 \mathrm{~B}$ mutant retinas (i.e., $U B C^{+/+} V h t^{f / 2 B}$ and $U B C^{C r e E R /+} V h t^{f / 2 B}$ ) had significant elevations in branching complexity compared with littermate controls (i.e., $U B C^{+/+} V h f^{f / f f}$ ) (Figure 1). Interestingly, retinal vessels in $V h l$ heterozygous $\left(U B C^{+/+} V h t^{t / 2 B}\right)$ mice exhibited a significant increase in arterial, but not venous, branching. Consistent with the $V h l^{-/-}$ES cell-derived vessels, the induced that $V h l$-null retinas (i.e., $U B C^{C r e E R /+} V h l^{f / f}$ ) lacked vessel-branching changes. Furthermore, conditional type 2B mutant vessels ( $V h l$ type $2 \mathrm{~B}$ mutant; i.e., $U B C^{\mathrm{CreER} /+} V h t^{f / 2 B}$ ) were more limited in their expansion across the retinal surface, as were the $U B C^{+/+} V h t^{t / 2 B}$ and $U B C^{C r e E R /+} V h f^{l / f l}$ vasculature, though to a lesser extent. Arterial sprouting in littermate control retinas (i.e., $\left.U B C^{+/+} V h f^{f / f f}\right)$ was significantly (1.5-fold) higher than sprout formation within venous regions; however, this difference was lost with all induced $V h l$ mutations $(U B C$ -

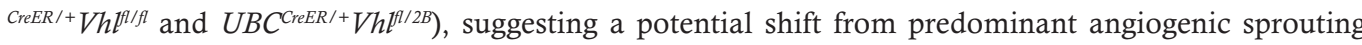
and moderate vessel maturation to a balance between these 2 stages of vessel formation. Overall, these data are consistent with the idea that the branching complexity of developing vessels is severely affected by the type 2B Vhl mutation, such that even in the presence of the WT Vhl gene, we observe disrupted vascular development ( $V h t^{1 / 2 B}$ heterozygotes), while heterozygous or homozygous deletions of the $V h l$ gene are less disruptive to normal blood vessel formation. 

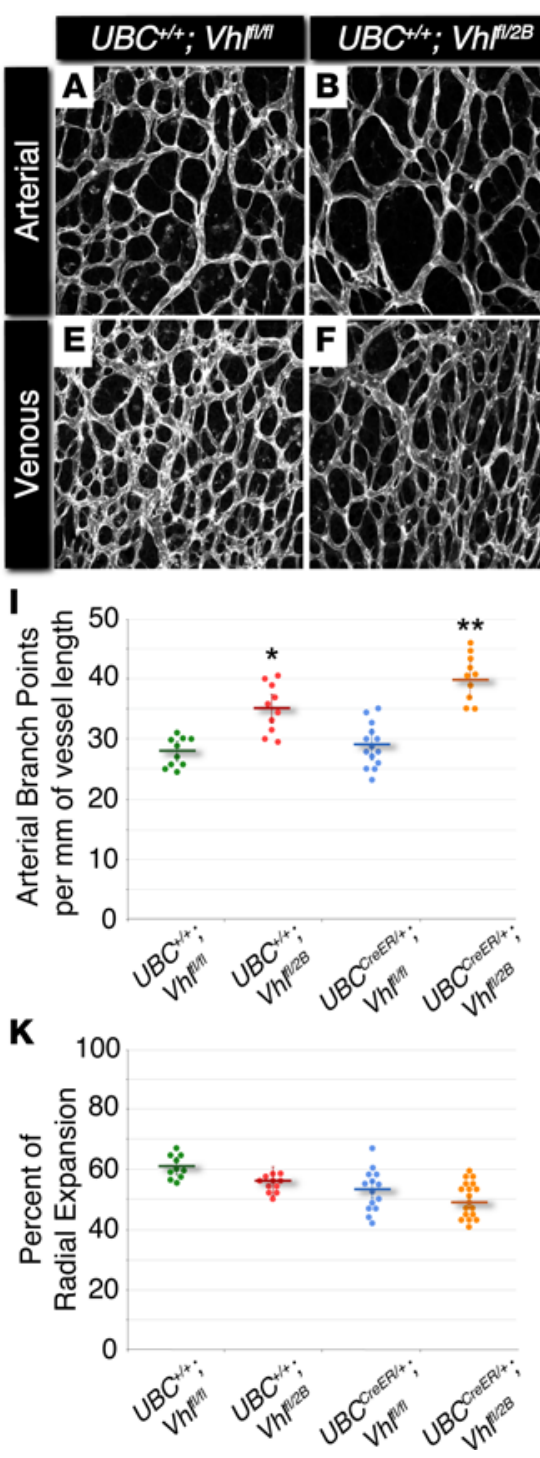
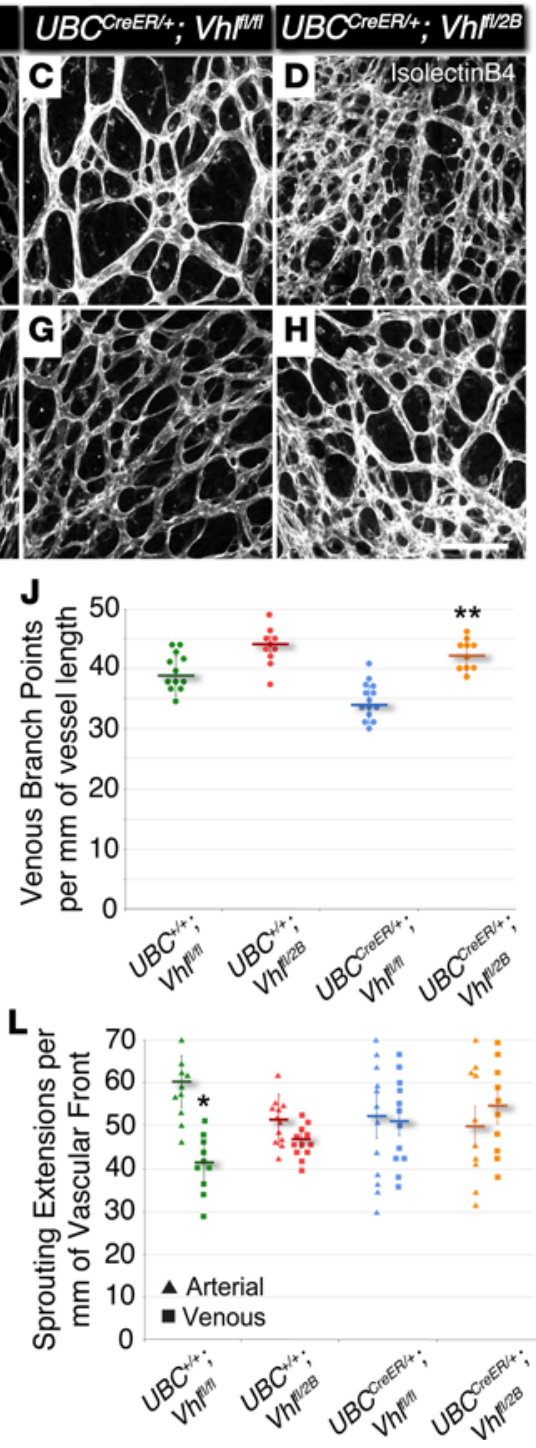

Figure 1. The Vhl 2B mutation causes increased arterial and venous branching in P5 mouse retinas without changes in vessel expansion or endothelial cell sprouting. (A-H) Representative images of P5 mouse retinal vasculature stained with isolectin $B 4$ Arterial (A-D) and venous (E-H) regions are shown for $U B C^{+/+} V h f^{f / / f l}, U B C^{+/+} V h l^{f / 2 B}, U B C^{\text {CreER/++} V h / f / / f l, ~ a n d ~}$ $U B C^{\text {CreER/+}} V h I^{f / 2 B}$ littermates exposed to tamoxifen. Scale bar: $100 \mu \mathrm{m}$. (I) Arterial branch points per vessel length measured from $\mathrm{P5}$ vascular networks. ${ }^{*} P \leq$ 0.05 vs. $U B C^{+++} V h l^{f / / f l} .{ }^{* *} P \leq 0.01$ vs. $U B C^{+++} V h l^{f / / f l}$ and $U B C^{\text {CreER/+} V h / f / f l}$. (J) Venous branch points per vessel length measured from P5 vascular networks. ${ }^{*} P \leq$

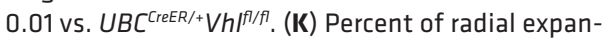
sion of retinal vessels from the optic disc toward the peripheral edge. No significant differences found. (L) Endothelial sprouting extensions per vessel length of the vascular front for arterial (triangles) and venous (squares) regions. ${ }^{*} P \leq 0.05 \mathrm{vs}$. $U B C^{+/+} \mathrm{Vh}^{f / / f l}$ arterial. All others are not significantly different. Values are averages \pm SEM. $n=10-15$ retina images per group (i.e., $2-3$ from 5 experimental litters). All statistical comparisons performed using 1-way ANOVA followed by pair-wise comparisons with a 2-tailed Student $t$ test.

Notch inhibition rescues maturation defects in the retinal vasculature of postnatal Vhl mutant mice. Vessel-branching irregularities observed in the development of conditional Vhl mutant retinas suggested that additional aspects of arterial and venous network formation might also be perturbed. Because Notch signaling is known to be involved in arterial-venous specification and vessel maturation (16-18, 20), we hypothesized that later stages of vascular development might also be adversely affected by the induction of genetic Vhl mutations. Therefore, we analyzed the developing retinal vasculature of P7 conditional $\mathrm{Vhl}$ mutant mice for branching complexity and hallmarks of arterial maturation, specifically the morphology and spatial distribution of $\alpha \mathrm{SMA}^{+}$vessels. In addition, we explored the vessel maturation role of Notch signaling downstream of $V h l$ mutations by pharmacologically blocking the Notch pathway in each of the Vhl mutant backgrounds (P5-P6 i.p. administration of the $\gamma$-secretase inhibitor DAPT; Supplemental Figure 2), aiming to observe a potential rescue in branching and arterial maturation defects. Vessel branching within arterial and venous regions was significantly reduced in conditional $V h l$-null retinas ( $U B C^{C r e E R /+} V h f^{l / f l}$; Figure 2), comparable with the sparsely branched retinal vasculature of mice with a gain-of-function mutation for Notch signaling (i.e., conditional overexpression of Notch1 intracellular domain [N1ICD], $U B C^{C r e E R /+}{ }^{2} o s a^{N I I C D}$; Supplemental Figure 3). In contrast, induction of the type 2B $V h l$ mutation $\left(U B C^{C r e E R /+} V h t^{I / 2 B}\right.$ ) had little to no effect on branching (Figure 2). Notch inhibition elevated retinal vessel branching within all experimental groups, regardless of association with arterial and venous areas. In spite of these increases, $U B C^{C r e E R /+} V h t^{f / f l}$ vasculature was not fully rescued back to littermate control branching levels by blocking Notch signaling. These observations 


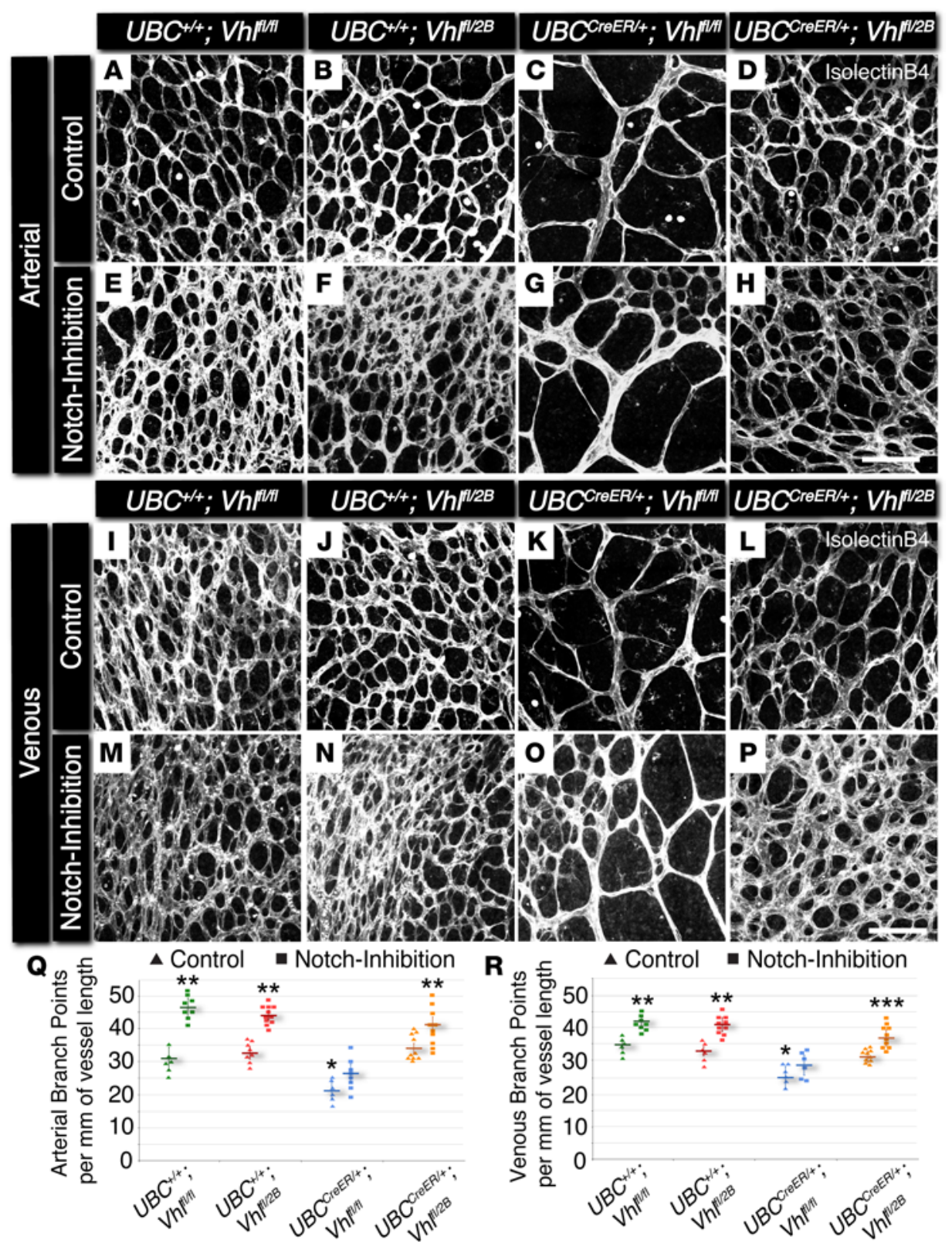

Figure 2. Conditional loss of $\mathbf{V h l}$ in P7 mouse retinas yields a reduction in arterial and venous branching, which is partially rescued with Notch inhibition by DAPT. (A-P) Representative images of P7 mouse retinal vasculature stained with isolectin B4. Arterial (A-H) and venous (I-P) regions are shown for $U B C^{+/+} V h l^{f / f l}$, $U B C^{+/+} V h f^{f / 2 B}, U B C^{C r e E R /+} V h l^{f l / f l}$, and $U B C^{C r e E R /+} V h I^{f / 2 B}$ mutant littermates exposed to tamoxifen and subjected to either control treatment (DMSO) or Notch inhibition (DAPT). Scale bars: $100 \mu \mathrm{m}$. (Q) Arterial branch points per vessel length measured from control (triangles) and Notch-inhibited (squares) P7 vascular networks. ${ }^{*} P \leq 0.05$ vs. control $U B C^{+/+} V h l-$ ${ }^{f l / 2 B}$ and $U B C^{C r e E R /+} V h l^{f / 2 B}$. ${ }^{*} P \leq 0.05$ vs. control within the same genotype, and vs. Notch-inhibited UBC $C^{\mathrm{Cr} E R /+} V h I^{f / / f l}$.

(R) Venous branch points per vessel length measured from control (triangles) and Notch-inhibited (squares) P7 vascular networks. ${ }^{*} P \leq 0.05$ vs. control $U B C^{+/+} V h f^{f / f l}$ and $U B C^{+/+} V h I^{f / 2 B}$. ${ }^{*} P \leq 0.05$ vs. control within the same genotype, and vs. Notch-inhibited UBC[reER/+$V h f^{f / f l}$. ${ }^{* * *} P$ $\leq 0.05$ vs. $U B C^{C r e E R /+} V h l^{f / 2 B}$. Values are averages \pm SEM. $n=6-8$ retina images per group (i.e., 3-4 from 2 experimental litters). All statistical comparisons performed using 1-way ANOVA followed by pair-wise comparisons with a 2-tailed Student $t$ test.

again underscore that the type $2 \mathrm{~B} V h l$ mutation uniquely affects blood vessel formation, consistent with the previously described data from the ES cell-derived vessels and the P5 retinal vasculature.

To compare the influence of the different $V h l$ mutations on vessel maturation, we assessed P7 retinal vasculature for the morphology and spatial distribution of $\alpha \mathrm{SMA}^{+}$vessels. Specifically, we measured (i) the percent length of primary arterial vessels covered with cells expressing $\alpha \mathrm{SMA}$, (ii) the diameter of these primary retinal arteries/arterioles, and (iii) the relative amount of $\alpha$ SMA expression aberrantly occurring beyond the primary retinal arteries (i.e., within the capillary plexus region at the vascular front) (Figure 3). In contrast to vessel-branching changes, arterial morphologies were perturbed in both induced $V h l$-null and -type $2 \mathrm{~B}$ mutations ( $U B C^{C r E R /+} V h f^{A / f l}$ and $U B C^{C r E R R /+} V h f^{f / 2 B}$, respectively). Primary arteries within these $\mathrm{Vhl}$ mutant retinas displayed $\alpha \mathrm{SMA}$ expression extending further toward the vascular front, and these arteries had significantly larger diameters compared with the littermate control retinas (both $U B C^{+/+} V h l^{\text {Il/fl}}$ and $U B C^{+/+} V h f^{t / 2 B}$ ). Strikingly, $\alpha$ SMA expression along microvessels at the periphery of the $V h l$-null and -type 2B mutant retinal vasculature exhibited an abnormal spatial distribution. In particular, more perivascular cells at the vascular front expressed a higher level of $\alpha$ SMA than is typically displayed at this developmental time point (21), and this excess of $\alpha$ SMA was not found in nonmutant littermate controls harboring at least 

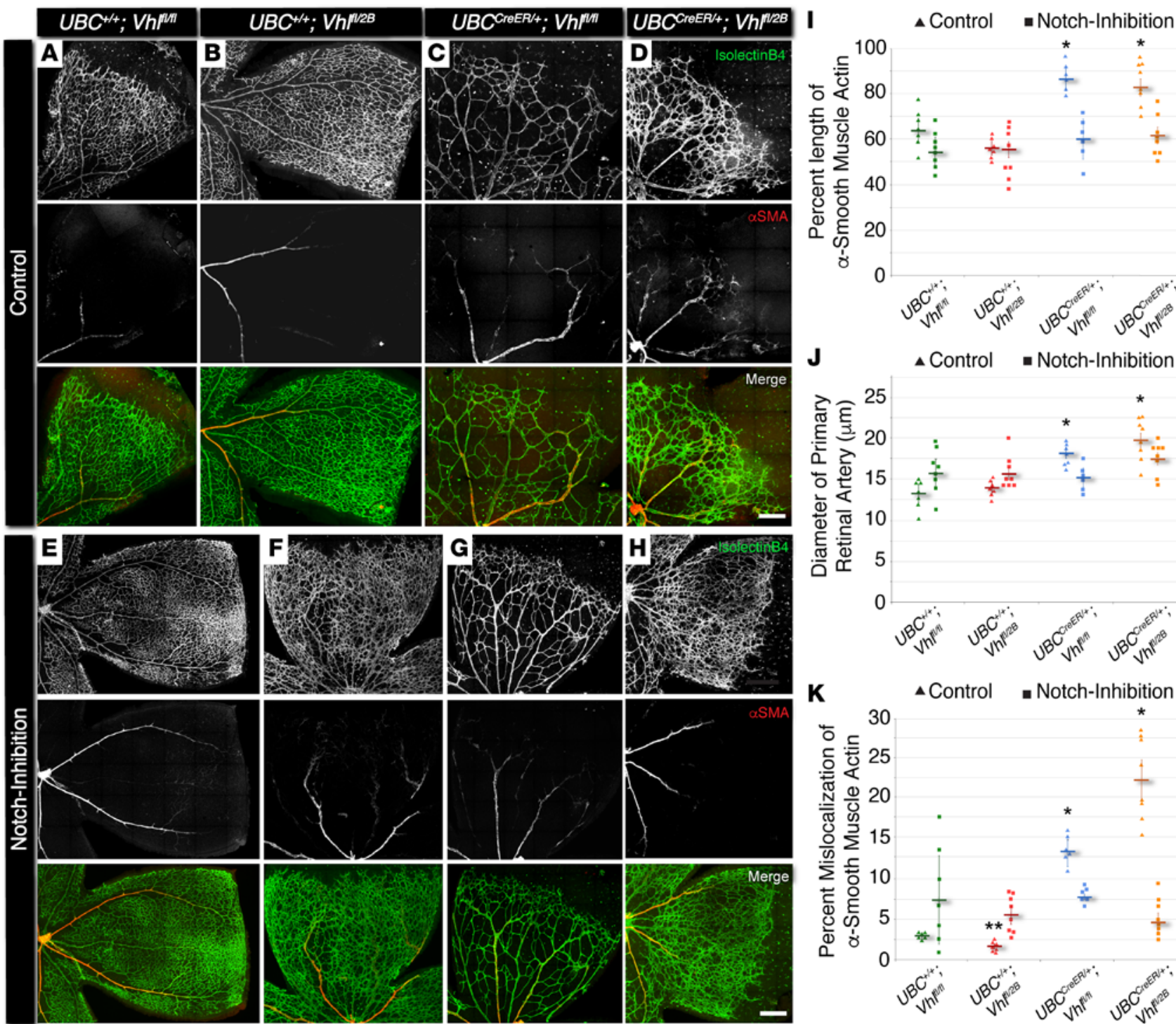

A Control - Notch-Inhibition
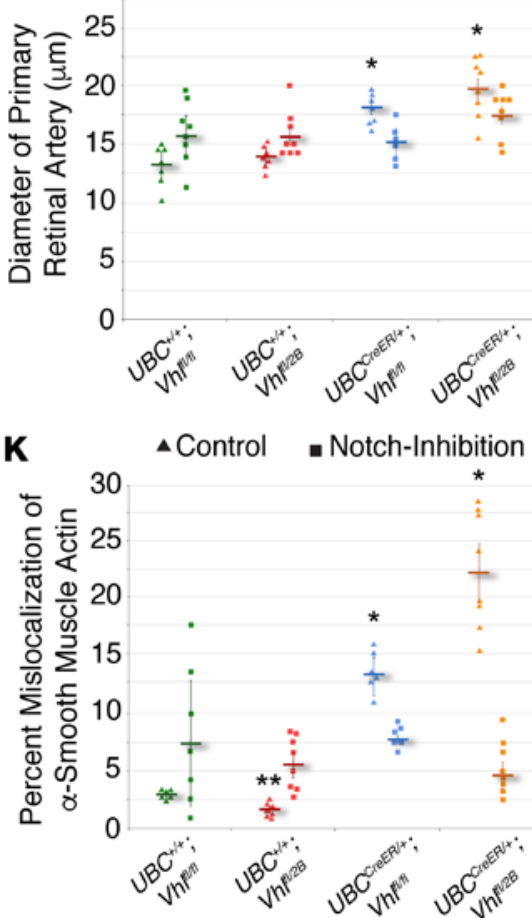

Figure 3. In P7 mouse retina, conditional Vhl-null and -type 2B mutations accelerate vessel maturation via excess alpha-smooth muscle actin expression, and this defect can be rescued by blocking Notch signaling. (A-H) Representative images of P7 mouse retinal vasculature stained with

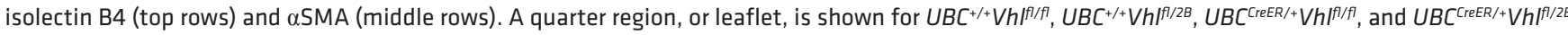
littermate retinas exposed to tamoxifen and subjected to either control treatment (DMSO, A-D) or Notch inhibition (DAPT, E-H). Scale bars: $200 \mu \mathrm{m}$. (I) Percent length of primary artery covered by $\alpha \mathrm{SMA}^{+}$cells in control (triangles) and Notch-inhibited (squares) P7 vascular networks. ${ }^{*} P \leq 0.05$ vs. control $U B C^{+/+} V h^{f / / f l}$ and $U B C^{+/+} V h h^{f / 2 B}$, and vs. Notch-inhibited within same genotype. (J) Diameter of primary retinal artery measured from control (triangles) and Notch-inhibited (squares) P7 vascular networks. ${ }^{*} P \leq 0.05$ vs. control $U B C^{+/+} V h f^{f / f l}$ and $U B C^{+/+} V h f^{f / 2 B}$, and vs. Notch-inhibited within same genotype. (K) Percent mislocalization of $\alpha$ SMA expression not associated with primary retinal arteries in control (triangles) and Notch-inhibited (squares) retinas. ${ }^{*} P \leq$

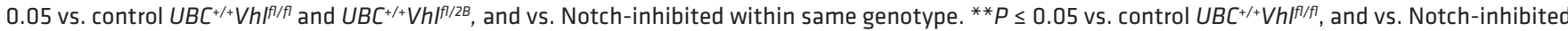
$U B C^{+/+} V h^{f / 2 B}$. Values are averages \pm SEM. $n=6-8$ retina images per group (i.e., 3-4 from 2 experimental litters). All statistical comparisons performed using 1-way ANOVA followed by pair-wise comparisons with a 2-tailed Student $t$ test.

$1 \mathrm{WT} V h l$ allele (i.e., $U B C^{+/+} V h h^{1 / f l}$ and $U B C^{+/+} V h t^{1 / 2 B}$ ) (Figure 3). Notch inhibition rescued all of the defects observed in the morphology and spatial distribution of $\alpha \mathrm{SMA}^{+}$vessels of $\mathrm{P} 7$ retinal vasculature. Taken together, these results, which are summarized in Supplemental Figure 4, suggest that Notch signaling makes critical contributions to developmental maturation of arterial vessels in the setting of $V h l$ deficiency.

Vascular pericytes have been suggested to regulate angiogenic remodeling at the retinal vascular front, specifically by influencing endothelial cell interactions via Notch signaling (30). In addition, recent studies have suggested that coronary SMCs can arise during development from pericytes expressing neural glial antigen 2 (NG2) (31). Thus, we asked if the perivascular cells with increased $\alpha$ SMA expression in 

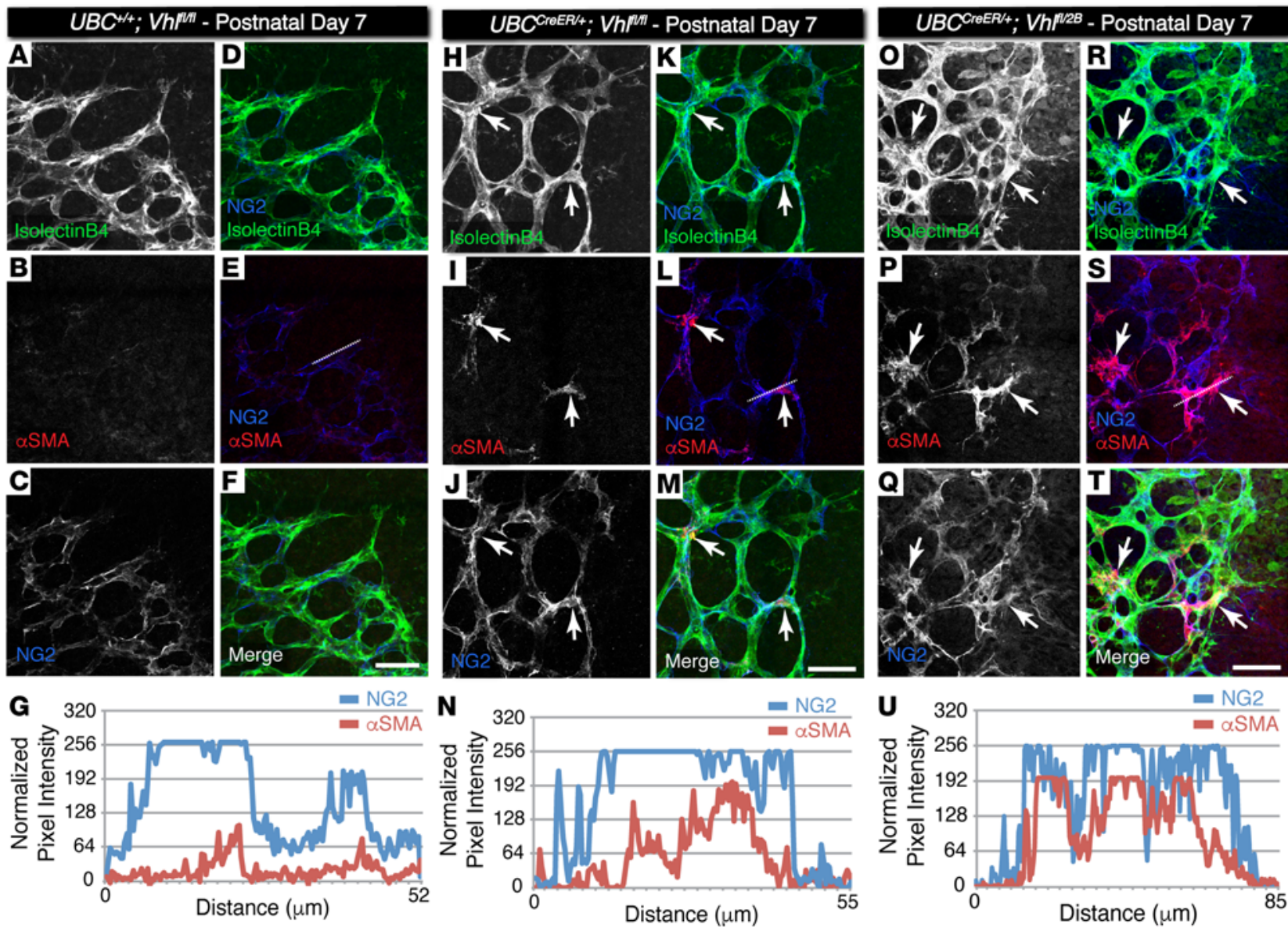

Figure 4. Pericytes at the vascular front of P7 conditional Vhl-null and Vhl type 2B mutant retina vessel networks ectopically express $\alpha$ SMA but not in littermate control vessels. (A-F, H-M, O-T) Representative images of P7 mouse retinal vasculature stained for isolectin B4 (A, H, O), $\alpha$ SMA (B, I, P), and neural glial antigen-2 (NG2) (C, J, Q). The NG2 image is merged with the isolectin B4 image (D, K, R), with the $\alpha$ SMA image (E, L, S), and with both isolectin $\mathrm{B} 4$ and $\alpha \mathrm{SMA}(\mathbf{F}, \mathbf{M}, \mathbf{T})$. Arrows indicate $\mathrm{NG2}^{+}$cells overlapping with $\alpha \mathrm{SMA}$ signal. The dotted lines in $\mathbf{E}$, $\mathbf{L}$, and $\mathbf{S}$ indicate the region of interest where pixel intensity values were measured and normalized. Scale bars: $50 \mu \mathrm{m}$. (G, N, U) Pixel intensity values for each line scan of NG2 and $\alpha$ SMA signals in the region indicated by the dotted line drawn in $\mathbf{E}, \mathbf{L}$, and $\mathbf{S}$, respectively.

the conditional $V h l$ mutant retinas were also positive for NG2, a well-established retinal pericyte marker (32). Indeed, we found a substantial overlap in the $\alpha$ SMA and NG2 signals (Figure 4), suggesting that the genetic loss of the $V h l$ gene $\left(U B C^{C r e E R /+} V h f^{f / f f}\right)$ and, to a greater extent, the type 2B $V h l$ mutation $\left(U B C^{C r e E R /+} V h l^{t / 2 B}\right)$ accelerates vascular pericyte expression of $\alpha$ SMA that likely yields an increased population of contractile vascular SMCs.

Notch signaling is among several vascular morphogenesis pathways disrupted by inducing Vhl loss or type $2 B$ mutation. The pVHL regulates HIF activity, which in turn modulates numerous downstream vascular morphogenesis cues including VEGF-A and PDGF, among several others (8). While our data support Notch signaling as an additional downstream effector of pVHL activity, we hypothesized that the Vhl-null and -type $2 \mathrm{~B}$ mutation scenarios (i.e., $U B C^{C r e E R /+} V h t^{l / f l}$ and $U B C^{C r e E R /+} V h l^{f / 2 B}$, respectively) likely elicit critical changes in gene expression within other vascular morphogenesis pathways, in addition to Notch. To test this idea, we performed transcriptome analysis of whole retinas from $\mathrm{P} 7$ conditional $\mathrm{Vhl}$-null and -type 2B mutation mice (and control littermates). RNA-Seq data (uploaded to NCBI Gene Expression Omnibus; GEO) was mapped to the mouse reference genome, and we conducted pathway analysis using the Database for Annotation, Visualization and Integrated Discovery (DAVID) $(33,34)$. In addition to significant changes in the expression of Hey2, an important transcriptional target in the Notch pathway (Figure 5), we found disrupted transcription of components within other pathways as well. Among several effectors altered within the HIF pathway, Egl-9 Family Hypoxia Inducible Factor $3(\mathrm{Eg} \ln 3)$ was upregulated in the null $\left(U B C^{C r e E R /+} V h f^{f / f f}\right)$ and type $2 B$ $\left(U B C^{C r E R /+} V h t^{I / 2 B}\right)$ retinas, consistent with VHL/HIF signaling dynamics in hypoxia (35). Also significantly 
A

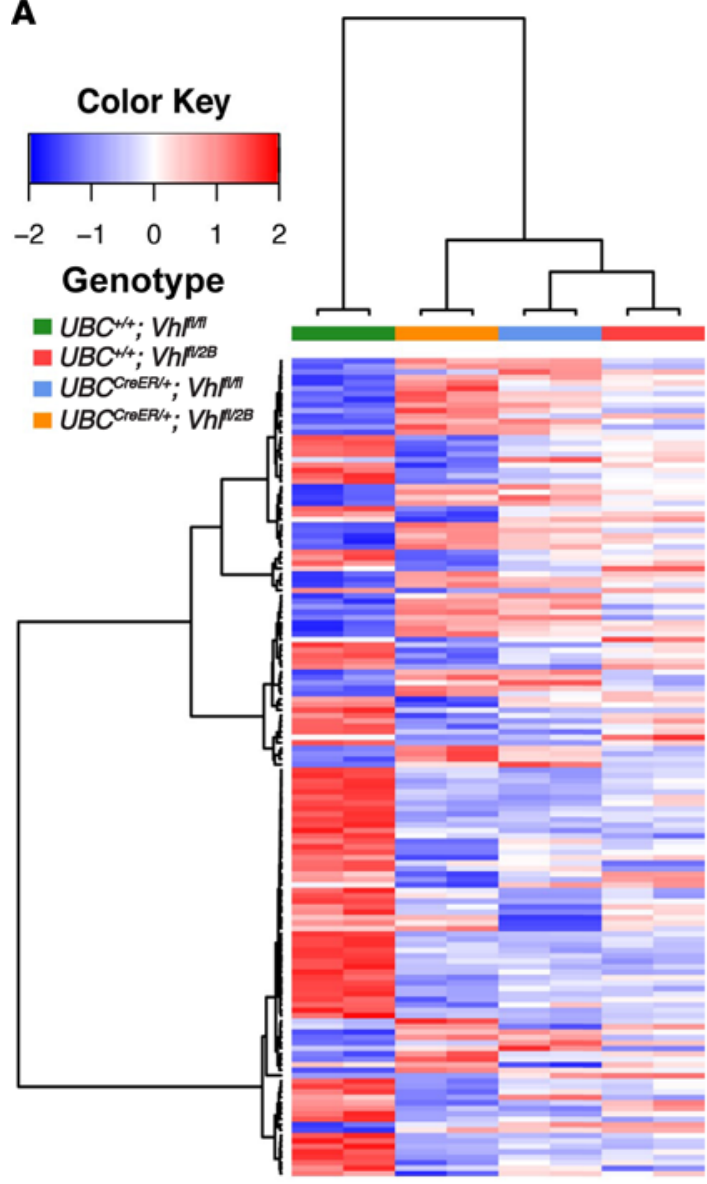

B

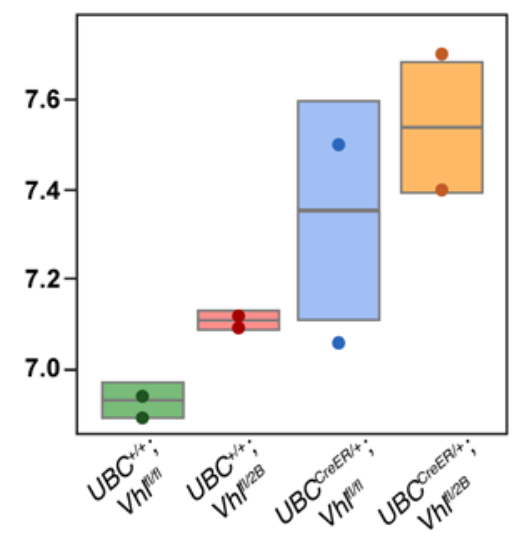

Arhgef12

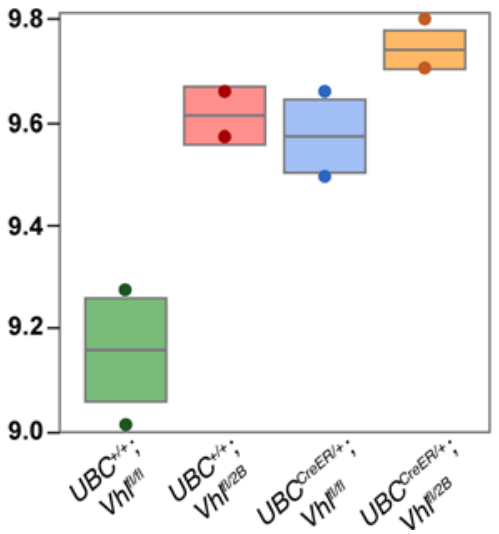

C

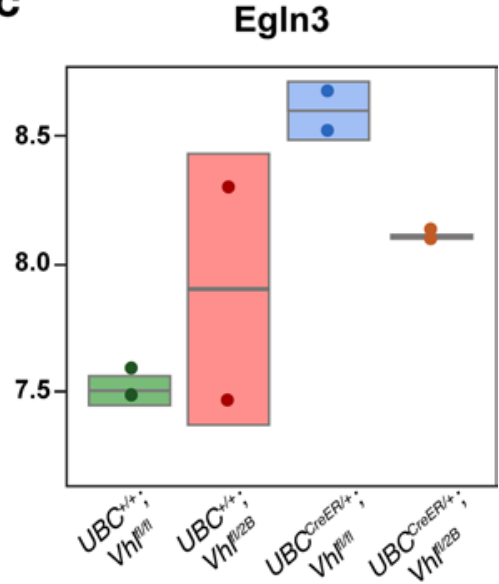

E

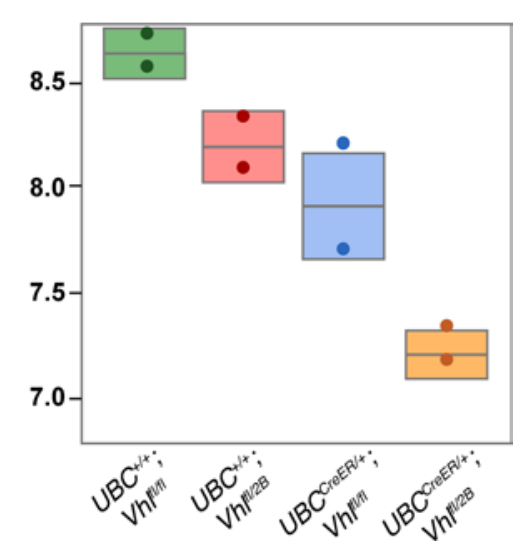

Figure 5. RNA-Seq analysis of P7 conditional Vhl-null and VhI-2B mutant retinas identifies expression changes in the Notch, hypoxia-inducible factor (HIF), smooth muscle contraction, and FoxO/TGF $\boldsymbol{\beta}$ pathways, among others. (A) Heatmap of the 200 most differentially regulated genes for each of the 4 groups: $U B C^{+/+} V h l^{f / f l}$ (green), $U B C^{+/+} V h f^{f / 2 B}$ (red), $U B C^{C r e E R /+} V h f^{f / f l}$ (blue), and $U B C^{C r e E R /+} V h I^{f / 2 B}$ (orange). Color key indicates relative levels of gene expression changes, with darker blue indicating downregulation and darker red indicating upregulation. (B-E) Box plots for representative genes within each pathway with substantial changes: (B) Hey2 (Notch), (C) Egln3 (HIF), (D) Arhgef12 (smooth muscle contraction), and (E) Gadd45b (FoxO/TCF $\beta$ ).

elevated was the expression of genes involved in vascular smooth muscle contraction, such as for rho guanine nucleotide exchange factor 12 (Arhgef12), which contributes to myosin regulation in SMCs (36). Transcriptional regulation within the FoxO/TGF $\beta$ signaling axis was also severely affected, as seen in reduced expression of Gadd45b. This protein induces growth arrest and can limit downstream TGF $\beta$ signaling (37); thus, reduced Gadd45b expression in the Vhl-null and -type 2B backgrounds suggests a lack of cell growth arrest and a permissive environment for TGF $\beta$ signaling, which is known to promote SMC differentiation $(38,39)$. These transcriptional changes, alongside our observation of accelerated arterialization of the retinal vasculature in $V h l$-null and -type $2 \mathrm{~B}$ conditional mutants $\left(U B C^{C r e E R /+} V h t^{l / f l}\right.$ and $\left.U B C^{C r e E R /+} V h f^{7 / 2 B}\right)$, lend further support to the idea that these $V h l$ mutations contribute to vascular defects encompassing misregulation of angiogenic remodeling, as well as of vessel maturation.

Retinal blood vessel defects in Vhl-null and-type $2 B$ mutant mice worsen by $P 21$. To determine if the vascular defects in the $V h l$ genetic loss and -type $2 \mathrm{~B}$ mutation mice ( $U B C^{C r e E R /+} V h l^{f l f l}$ and $U B C^{C r e E R /+} V h t^{f / 2 B}$ ) persisted beyond early postnatal stages, we collected retinas from P21 mice (weaning) following the induction of these mutations from P1-P3. We found gross vascular dysmorphogenesis in the Vhlnull and -type $2 \mathrm{~B}$ mutant retinas, including vessel patterning defects and extensive mis-localization of $\alpha$ SMA along retinal arteries and veins (Figure 6). Type $2 \mathrm{~B}$ mutant vasculature $\left(U B C^{\mathrm{CreER} /+} V h \mathrm{fl}^{f / 2 B}\right.$ ) appeared most severely affected, as these retinal vessels were more randomly distributed and exhibited aberrant $\alpha$ SMA expression along smaller diameter arterioles and arterial branches that connected directly to venules. While our conditional $V h l$ loss and -type 2B mutation models do not replicate 



Figure 6. Conditional Vhl-null and -type 2B mutations induce gross vascular dysmorphogenesis and severe mislocalization of aSMA in P21 mouse retinas. (A-D) Representative images of P21 mouse retinal vasculature stained with isolectin B4 (top row) and $\alpha$ SMA (middle row). An artery-vein pair is shown for $U B C^{+/+} V h^{f / / f l}(\mathbf{A}), U B C^{+/+} V h^{f / / 2 B}$ (B), $U B C^{C \text { reER/+}} V h^{f^{f / f}}(\mathbf{C})$, and $U B C^{C r e E R /+} V h l^{f / 2 B}$ (D) littermate retinas exposed to tamoxifen. Scale bars: $100 \mu \mathrm{m}$.

the full extent of the clinical situation, the vessel-branching and maturation phenotypes from the mouse data overlap with certain aspects of VHL-related retinopathy in patients (our unpublished data), warranting further investigation into how these vascular defects may give rise to clinical features of VHL-related retinopathy such as retinal capillary hemangiomas, exudative/tractional detachment, and scar tissue formation. Furthermore, they also support a model in which lesions in the VHL gene give rise to distinct vascular pathologies by disrupting vessel patterning mechanisms prior to the development of hemangioblastomas, such as those governed by the Notch pathway as well as those identified from our transcriptome analysis.

\section{Discussion}

VHL mutations cause defective blood vessel formation, as is often seen in the clinical presentation of hemangioblastomas within neural tissue of affected patients. In the present study, we show that conditional genetic loss of $\operatorname{Vhl}\left(U B C^{\mathrm{CreER} /+} V h l^{7 / f f}\right)$ and the type $2 \mathrm{~B} V h l$ mutation $\left(U B C^{\mathrm{CreER} /+} V h \mathrm{fl}^{1 / 2 B}\right)$ have differential effects on vascular development via downstream perturbations in Notch pathway signaling and in conjunction with other pathway misregulation. Endothelial Notch signaling plays a critical role in sprouting angiogenesis (10-12), in vessel maturation through arterial-venous specification (16), and in the induction of contractile vascular SMC differentiation $(17,18,20,21)$. Our observations suggest $V h l$ lesions disrupt Notch signaling such that processes underlying vessel maturation are accelerated; most prominent among these 
is an altered spatiotemporal expression of $\alpha \mathrm{SMA}$. The early stages of vessel formation and branching are compromised during $V h l$ loss $\left(U B C^{\mathrm{CreER} /+} V h l^{\mathrm{llf} f}\right)$ but not with the type $2 \mathrm{~B} V h l$ mutation $\left(U B C^{\mathrm{CreER} /+} V h l^{7 / 2 B}\right)$. These data suggest that $V h l$ mutations may not only cause substantial changes in angiogenic branching dynamics, but also strongly influence how blood vessels mature.

The pVHL provides essential regulation of HIFs, which in turn modulate downstream signals that promote vessel growth and remodeling such as VEGF-A. Kurihara et al. explored the VHL/HIF/VEGF signaling axis in postnatal retinas of mice with conditional $V h l$ loss (25). To induce cre/ERT2 expression in the retina, the authors used the Pax6 promoter, which is present in retina neural progenitor cells but may also be expressed by endothelial cells during early neural tissue development (40). Interestingly, they found that conditional Vhl-KO induced ectopic VEGF-A expression, but retinal blood vessel branching actually decreased, as was observed in the present study. Kurihara and colleagues found that diminished vascular branching resulted largely from vessel regression, which likely represents the vessel pruning phase of retina vascular maturation (41). In contrast, the conditional type $2 \mathrm{~B} V h l$ mutation generated in the current study (i.e., $U B C^{\mathrm{CreER} /+} V h t^{f / 2 B}$ ) did not cause a decrease in network complexity, and arterial branching was in fact enhanced significantly at P5. These results suggest that, while both Vhl genetic defects disrupt the vessel maturation phase, the type $2 \mathrm{~B}$ mutation has differential effects on the initial stages of vascular development compared with the null mutation. This disruption of both the early and later stages of vessel formation may contribute to more extensive, and perhaps aggressive, blood vessel remodeling defects in patients carrying the type $2 \mathrm{~B}$ mutation. Collectively, these data suggest that carriers of type $2 \mathrm{~B}$ mutations may already have or display some of these vascular defects and should be monitored closely, even in the absence of a "second-hit."

Misregulation of the Notch signaling pathway has been implicated as a key factor in VHL syndrome manifestations and, most notably, in ccRCC onset and progression. Recent studies have shown an upregulation of the Notch1 receptor in human ccRCC, as well as increased levels of the cleaved intracellular region of Notch1 responsible for downstream signaling (23). Expression of the Notch ligand Jag1 is also highly upregulated in human ccRCC cells (24). While these changes in the Notch pathway have been implicated as contributing factors to renal cell transformation and ccRCC pathogenesis, disruptions in Notch signaling within vascular tissue may also influence disease progression by exacerbating pathological blood vessel remodeling. Our observations suggest that $V h l$ mutations likely influence vascular patterning in a way that undermines the Notch pathway, among several other effector pathways, such that blood vessel maturation is accelerated toward an arterial phenotype. With the formation of larger-caliber vessels, which presumably enhance blood flow to downstream tissues, it is intriguing to speculate that these changes may be an important factor in the aggressive growth and metastatic potential of ccRCC tumors (42). Thus, targeting the Notch pathway may rescue oncogenic defects, such as cell cycle misregulation (24), while also limiting tumor growth and metastasis by restricting the expansion of tumor-associated arterial vessels.

In the developing retina, vascular pericytes ensheath newly formed blood vessels to promote their stability and integrity. Pericytes can also give rise to arterial SMCs via Jag1/Notch3 signaling, as shown recently in the developing coronary arteries (31). The contractile capacity of capillary pericytes remains an open question $(43,44)$; however, in the current study, we found ectopic $\alpha \mathrm{SMA}$ expression within NG2+ pericytes at the periphery of the vasculature within conditional $V h l$-null and -type $2 \mathrm{~B}$ mutants (i.e., $U B C^{\mathrm{CreER} /+} V h \mathrm{f}^{l / f l}$ and $U B C^{\mathrm{CreER} /+} V h t^{\mathrm{fl} / 2 B}$, respectively). Mural cells at the vascular front of littermate control retinas (i.e., $U B C^{+/+} V h t^{7 / / 1}$ and $U B C^{+/+} V h t^{1 / 2 B}$ ) lacked $\alpha$ SMA expression at this time point. This aberrant expression of $\alpha \mathrm{SMA}$ in $V h l$ mutant animals suggests an accelerated maturation of retinal pericytes, presumably into precursors for SMCs. The differential effects of $V h l$-null and -type 2B mutant $V h l$ retinal models are particularly interesting in light of the broad range of phenotype in patients with VHL syndrome with regard to retinal involvement and disease severity. Notch inhibition via DAPT treatment rescued this phenotypic abnormality, consistent with the notion that the $V h l$ mutations augment Notch pathway signals underlying SMC differentiation $(17,18,20,21)$. Notch signaling perturbations likely compound, and potentially intersect with, other cues downstream of pVHL activity, such as PDGF-BB (45) and TGF $\beta$ (39), both of which are known to promote SMC differentiation. Future work will be needed to extend the pathway analysis from the conditional $\mathrm{Vhl}$ null and-type 2B mutant transcriptional profiles to elucidate the crosstalk between the Notch pathway and other signaling axes involved in the observed vascular dysmorphogenesis. Nevertheless, our results therefore implicate the Notch pathway as a potential therapeutic target for treating not only early-stage vascular malformations in patients with VHL syndrome, but also those defects affecting blood vessel maturation. 
Deletion and type 2B VHL mutations underlie an increased risk for hemangioblastoma and ccRCC formation, owing in part to altered HIF expression (1) as well as defects in numerous other downstream pathways. While both VHL mutations cause abnormal, and often excessive, blood vessel remodeling in the clinical setting, our data suggest that these genetic lesions likely have differential effects on the nature of these vascular changes. Vhl-null mutation $\left(U B C^{C r e E R /+} V h t^{f / f f}\right)$ led mostly to accelerated vessel maturation, while the type 2B $V h l$ mutation $\left(U B C^{C r e E R /+} V h f^{t / 2 B}\right)$ caused an additional increase in vessel-branching complexity. The previously described partial regulation of HIFs by the mutant 2B Vhl (7) likely gives rise to a unique VHL/ HIF downstream gene target axes affecting the aberrant vascular formation observed in type $2 \mathrm{~B}$ mutants not commensurate in the $\mathrm{Vhl}$-null setting. These results also may help explain the heterogeneity observed in the vascular architecture and integrity within ccRCC tumors (46), as well as the apparent discrepancy between levels of angiogenic factors, tumor vessel content, and tumor growth (28). This disconnect between proangiogenic signals and vessel outcomes has been linked to disruptions in the Notch pathway $(14,47)$. Our data also strongly implicate compromised Notch signaling downstream of $V h l$ mutations as a prominent molecular effector for the vascular dysmorphogenesis observed. Thus, developing therapeutic targets within the Notch pathway could offer novel treatment options for patients with VHL syndrome, especially those suffering from recurrent hemangioblastoma formation and ccRCC.

\section{Methods}

Mutant mice and inducible genetic recombination experiments. Mice expressing Cre-recombinase ubiquitously (Tg[UBC-cre/ERT2], The Jackson Laboratory, catalog 007001; denoted in the current study as UBC CreER/+ with $C r e E R^{-}$animals denoted as $U B C^{+/+}$) were bred with $V h t^{\text {tlfl }}$ mice (gift from Volker Haase, Vanderbilt University Medical Center; ref. 48). $V h l^{2 B /+}$ mice generated previously (4) were also crossed with $V h t^{\text {ft/fl }}$ mice to yield $V h t^{\pi / 2 B}$ offspring. Breeding $U B C^{\text {CreER/+}} V h t^{f / f l}$ males with $V h t^{f / 2 B}$ females produced experimental litters with genotypes: (i) nonmutant littermate control, $U B C^{+/+} V h f^{l / f l}$; (ii) littermate control with 1 WT $V h l$ allele and one type 2B mutant $V h l$ allele, $U B C^{+/+} V h l^{7 / 2 B}$; (iii) conditional $V h l$-null, $U B C^{C r e E R /+} V h t^{1 / f f}$; and (iv) conditional $V h l$ type $2 \mathrm{~B}$ mutant, $U B C^{\mathrm{CreER} /+} V h t^{f / 2 B}$. Where possible, animals were distributed across sex to avoid the introduction of sex biasing. Pups were i.p. injected each day with $1 \mathrm{mg} / \mathrm{ml}$ of tamoxifen (Thermo Fisher Scientific) from P1-P3. Retinal harvest occurred on P5, P7, and P21 (Supplemental Figure 2). Cre-recombination activity following tamoxifen exposure was verified using a Cre reporter mouse line (Supplemental Figure 5). For Notch inhibition experiments, DAPT (Calbiochem) was dissolved in DMSO (MilliporeSigma), and $50 \mathrm{mg}$ per $\mathrm{kg}$ of animal weight was administered i.p. on P5 and P6, with retina harvest occurring on P7 (Supplemental Figure 2). Equivalent volumes of DMSO-only were injected at the same time points for vehicle control animals.

Retina immunostaining, imaging, and analysis. Eyes were fixed by whole animal perfusion with $0.5 \%$ paraformaldehyde (PFA; Electron Microscopy Services) followed by immersion in 2\% PFA for 2 hours at room temperature. Following PBS (VWR) rinse, retinas were dissected from the whole eye, immersed in 100\% cold ethanol for 30 minutes, rinsed with PBS containing 1\% Triton X-100 (PBS-T; Thermo Fisher Scientific) for 30 minutes at room temperature, and then blocked in 3\% donkey serum (Jackson ImmunoResearch) in PBS-T for 1 hour at room temperature. Primary antibody incubation was performed in PBS-T overnight at $4^{\circ} \mathrm{C}$ with isolectin GS-IB4 conjugated to AlexaFluor 488 (Molecular Probes, I21411, 1:100), aSMA conjugated to Cy3 (clone 1A4, MilliporeSigma, C6198, 1:200), and NG2 (MilliporeSigma, AB5320, 1:200). Secondary antibody incubation was also in PBS-T overnight at $4^{\circ} \mathrm{C}$ with donkey anti-rabbit AlexaFluor 647 (Jackson ImmunoResearch, 711-605-152, 1:400). Retinas were washed for 5 minutes 3 times in PBS-T, flattened, and mounted on slides in 50:50 PBS/glycerol (MP Biomedicals) with 1.5 coverslips. Images of retina whole mounts were acquired with a Zeiss LSM 880 confocal microscope with a $\times 40$ objective, and 10-15 z-axis confocal scans were acquired and compressed. ImageJ was used to measure: (i) vessel-branch point densities, as described in the Supplemental Methods; (ii) the percentage of the retina radial to the optic disc occupied by vasculature (i.e., radial expansion); (iii) the number of vessel sprouts per length of the vascular front; (iv) the percent length of the primary arteries covered by $\alpha \mathrm{SMA}^{+}$cells; (v) the diameters of primary retinal arteries; (vi) the percent of $\alpha \mathrm{SMA}^{+}$cells not associated with the primary artery (i.e., mislocalized); and (vii) pixel intensity values across a line region of interest (i.e., line scan tool) (Figure 4).

Retina transcriptome analysis. Following induction of the genetic mutations as described above, retinas were collected from P7 mice and immediately digested in TRIzol (Invitrogen) to extract mRNA transcripts ( $n=2$ for each genotype). RNA was further isolated with 1-Bromo-3-chloropropane (MilliporeSigma) and 
ethanol and purified with an RNeasy Plus Kit (Qiagen). Using an Agilent Bioanlayzer, the Virginia Tech Biocomplexity Institute ensured the isolated mRNA was of a sufficiently high quality for RNA-Seq. Eight samples (4 genotypes $\times 2$ replicates per genotype) were sent to GeneWiz for additional quality assessment, cDNA library generation, and sequencing on an Illumina HiSeq with a $2 \times 150$ configuration.

Sequencing reads were aligned against the Mouse GENCODE genome, Version M14 (January 2017 freeze, GRCm38, Ensemb1 89) using the Spliced Transcripts Alignment to a Reference (STAR) software $(49,50)$. Reads were preprocessed and index using SAMtools (51). Mapped reads were assigned to gene features and quantified using featureCounts (52). Normalization and differential expression was performed using DESeq2 (53). The top most significantly differentially expressed genes (FDR $<0.01$ and $\log _{2}$ difference greater than 0.5 in magnitude) were considered for subsequent functional enrichment using DAVID bioinformatics tools $(33,34)$. The top 200 most differentially expressed genes were used for unsupervised hierarchical cluster analysis and visualized using heatmap representations. Raw data files are available upon request or from NCBI GEO (accession number GSE109102).

Statistics. Statistical analysis was conducted using GraphPad Prism 6. Multiple measurements were made for each parameter, and an average and SD were calculated from these values. Statistical comparisons were made using 1-Way ANOVA followed by pair-wise comparisons by 2-tailed Student $t$ test. A P value less than 0.05 was considered significant.

Study approval. All animal experiments were conducted with review and approval from the UNC-CH and Virginia Tech IACUC. All protocols are reviewed and approved by IACUC boards. The UNC-CH NIH/PHS Animal Welfare Assurance Number is A3410-01 (expires 4/30/2021). The Virginia Tech NIH/ PHS Animal Welfare Assurance Number is A-32081-01 (expires: 7/31/2021).

\section{Author contributions}

Research studies were designed by AA, LBP, VLB, WKR, and JCC. AA and JCC conducted experiments, and AA, ST, LBP, MHJ, and JCC acquired the primary data. Data analysis was performed by AA, AADC, HZ, JD, and JCC. AA, VLB, WKR, ABD, and JCC prepared the manuscript.

\section{Acknowledgments}

We would like to thank members of the Rathmell, Bautch, and Chappell labs for discussions concerning the data and manuscript preparation. This work was supported by NIH grants R01HL43174 (to VLB), R21CA184387 (to WKR), K99HL105779, and R00HL105779 (both to JCC) and a NIH NRSA grant F31CA154080 (to AA).

Address correspondence to: John C. Chappell, Virginia Tech Carilion Research Institute, 2 Riverside Circle, Roanoke, Virginia 24016, USA. Phone: 540.526.2219; Email: JChappell@vtc.vt.edu. Or to: W. Kimryn Rathmell, Vanderbilt University, 798 Preston Research Building, 2220 Pierce Avenue, Nashville, Tennessee 37232-6307, USA. Phone: 615.936.3320; Email: Kimryn.Rathmell@Vanderbilt.edu.

AA's present address is: Department of Pathology and Laboratory Medicine McLendon Clinical Laboratories, UNC Hospital, Chapel Hill, North Carolina, USA.

1. Gossage L, Eisen T, Maher ER. VHL, the story of a tumour suppressor gene. Nat Rev Cancer. 2015;15(1):55-64.

2. Keefe SM, Nathanson KL, Rathmell WK. The molecular biology of renal cell carcinoma. Semin Oncol. 2013;40(4):421-428.

3. Clifford SC, et al. Contrasting effects on HIF-1alpha regulation by disease-causing pVHL mutations correlate with patterns of tumourigenesis in von Hippel-Lindau disease. Hum Mol Genet. 2001;10(10):1029-1038.

4. Lee CM, et al. VHL Type 2B gene mutation moderates HIF dosage in vitro and in vivo. Oncogene. 2009;28(14):1694-1705

5. Wind JJ, Lonser RR. Management of von Hippel-Lindau disease-associated CNS lesions. Expert Rev Neurother. 2011;11(10):1433-1441.

6. Kanno H, et al. Spinal cord hemangioblastomas in von Hippel-Lindau disease. Spinal Cord. 2009;47(6):447-452.

7. Hacker KE, Lee CM, Rathmell WK. VHL type 2B mutations retain VBC complex form and function. PLoS One. 2008;3(11):e3801.

8. Patel PH, Chadalavada RS, Chaganti RS, Motzer RJ. Targeting von Hippel-Lindau pathway in renal cell carcinoma. Clin Cancer Res. 2006;12(24):7215-7220.

9. Olsson AK, Dimberg A, Kreuger J, Claesson-Welsh L. VEGF receptor signalling - in control of vascular function. Nat Rev Mol Cell Biol. 2006;7(5):359-371.

10. Hellström M, et al. D114 signalling through Notch1 regulates formation of tip cells during angiogenesis. Nature. 2007;445(7129):776-780. 
11. Chappell JC, Mouillesseaux KP, Bautch VL. Flt-1 (vascular endothelial growth factor receptor-1) is essential for the vascular endothelial growth factor-Notch feedback loop during angiogenesis. Arterioscler Thromb Vasc Biol. 2013;33(8):1952-1959.

12. Suchting S, et al. The Notch ligand Delta-like 4 negatively regulates endothelial tip cell formation and vessel branching. Proc Natl Acad Sci USA. 2007;104(9):3225-3230.

13. Yan M, et al. Chronic DLL4 blockade induces vascular neoplasms. Nature. 2010;463(7282):E6-E7.

14. Ridgway J, et al. Inhibition of D114 signalling inhibits tumour growth by deregulating angiogenesis. Nature. 2006;444(7122):1083-1087.

15. Benedito R, et al. The notch ligands Dl14 and Jagged1 have opposing effects on angiogenesis. Cell. 2009;137(6):1124-1135

16. Hofmann JJ, Iruela-Arispe ML. Notch signaling in blood vessels: who is talking to whom about what? Circ Res. 2007;100(11):1556-1568.

17. High FA, Lu MM, Pear WS, Loomes KM, Kaestner KH, Epstein JA. Endothelial expression of the Notch ligand Jagged1 is required for vascular smooth muscle development. Proc Natl Acad Sci USA. 2008;105(6):1955-1959.

18. Pedrosa AR, et al. Endothelial Jagged1 antagonizes D114 regulation of endothelial branching and promotes vascular maturation downstream of D114/Notch1. Arterioscler Thromb Vasc Biol. 2015;35(5):1134-1146.

19. Lanner F, Sohl M, Farnebo F. Functional arterial and venous fate is determined by graded VEGF signaling and notch status during embryonic stem cell differentiation. Arterioscler Thromb Vasc Biol. 2007;27(3):487-493.

20. Liu H, Kennard S, Lilly B. NOTCH3 expression is induced in mural cells through an autoregulatory loop that requires endothelial-expressed JAGGED1. Circ Res. 2009;104(4):466-475.

21. Scheppke L, et al. Notch promotes vascular maturation by inducing integrin-mediated smooth muscle cell adhesion to the endothelial basement membrane. Blood. 2012;119(9):2149-2158.

22. Phng LK, Gerhardt H. Angiogenesis: a team effort coordinated by notch. Dev Cell. 2009;16(2):196-208

23. Johansson E, et al. Simultaneous targeted activation of Notch1 and Vhl-disruption in the kidney proximal epithelial tubular cells in mice. Sci Rep. 2016;6:30739.

24. Sjölund J, et al. Suppression of renal cell carcinoma growth by inhibition of Notch signaling in vitro and in vivo. J Clin Invest. 2008;118(1):217-228.

25. Kurihara T, et al. von Hippel-Lindau protein regulates transition from the fetal to the adult circulatory system in retina. Development. 2010;137(9):1563-1571.

26. Heath VL, Bicknell R. Anticancer strategies involving the vasculature. Nat Rev Clin Oncol. 2009;6(7):395-404.

27. Ribatti D. Tumor refractoriness to anti-VEGF therapy. Oncotarget. 2016;7(29):46668-46677.

28. Chappell JC, et al. Flt-1 (VEGFR-1) coordinates discrete stages of blood vessel formation. Cardiovasc Res. 2016;111(1):84-93.

29. Chappell JC, Taylor SM, Ferrara N, Bautch VL. Local guidance of emerging vessel sprouts requires soluble Flt-1. Dev Cell. 2009; 17(3):377-386

30. Walpole J, Mac Gabhann F, Peirce SM, Chappell JC. Agent-based computational model of retinal angiogenesis simulates microvascular network morphology as a function of pericyte coverage. Microcirculation. 2017;24(8).

31. Volz KS, et al. Pericytes are progenitors for coronary artery smooth muscle. Elife. 2015;4:e10036

32. Armulik A, Genové G, Betsholtz C. Pericytes: developmental, physiological, and pathological perspectives, problems, and promises. Dev Cell. 2011;21(2):193-215.

33. Huang da W, Sherman BT, Lempicki RA. Systematic and integrative analysis of large gene lists using DAVID bioinformatics resources. Nat Protoc. 2009;4(1):44-57.

34. Huang da W, Sherman BT, Lempicki RA. Bioinformatics enrichment tools: paths toward the comprehensive functional analysis of large gene lists. Nucleic Acids Res. 2009;37(1):1-13.

35. del Peso L, et al. The von Hippel Lindau/hypoxia-inducible factor (HIF) pathway regulates the transcription of the HIF-proline hydroxylase genes in response to low oxygen. J Biol Chem. 2003;278(49):48690-48695.

36. Somlyo AP, Somlyo AV. Ca2+ sensitivity of smooth muscle and nonmuscle myosin II: modulated by G proteins, kinases, and myosin phosphatase. Physiol Rev. 2003;83(4):1325-1358.

37. Balliet AG, Hollander MC, Fornace AJ, Hoffman B, Liebermann DA. Comparative analysis of the genetic structure and chromosomal mapping of the murine Gadd45g/CR6 gene. DNA Cell Biol. 2003;22(7):457-468.

38. Owens GK, Kumar MS, Wamhoff BR. Molecular regulation of vascular smooth muscle cell differentiation in development and disease. Physiol Rev. 2004;84(3):767-801.

39. Ananth S, et al. Transforming growth factor beta1 is a target for the von Hippel-Lindau tumor suppressor and a critical growth factor for clear cell renal carcinoma. Cancer Res. 1999;59(9):2210-2216.

40. Vasudevan A, Long JE, Crandall JE, Rubenstein JL, Bhide PG. Compartment-specific transcription factors orchestrate angiogenesis gradients in the embryonic brain. Nat Neurosci. 2008;11(4):429-439.

41. Korn C, Augustin HG. Mechanisms of Vessel Pruning and Regression. Dev Cell. 2015;34(1):5-17.

42. Sjölund J, et al. The notch and TGF- $\beta$ signaling pathways contribute to the aggressiveness of clear cell renal cell carcinoma. PLoS One. 2011;6(8):e23057.

43. Hill RA, Tong L, Yuan P, Murikinati S, Gupta S, Grutzendler J. Regional Blood Flow in the Normal and Ischemic Brain Is Controlled by Arteriolar Smooth Muscle Cell Contractility and Not by Capillary Pericytes. Neuron. 2015;87(1):95-110.

44. Hall CN, et al. Capillary pericytes regulate cerebral blood flow in health and disease. Nature. 2014;508(7494):55-60

45. Iliopoulos O, Levy AP, Jiang C, Kaelin WG, Goldberg MA. Negative regulation of hypoxia-inducible genes by the von Hippel-Lindau protein. Proc Natl Acad Sci USA. 1996;93(20):10595-10599.

46. Qian CN, Huang D, Wondergem B, Teh BT. Complexity of tumor vasculature in clear cell renal cell carcinoma. Cancer. 2009;115(10 Suppl):2282-2289.

47. Noguera-Troise I, et al. Blockade of D114 inhibits tumour growth by promoting non-productive angiogenesis. Nature. 2006;444(7122):1032-1037.

48. Haase VH, Glickman JN, Socolovsky M, Jaenisch R. Vascular tumors in livers with targeted inactivation of the von Hippel-Lindau tumor suppressor. Proc Natl Acad Sci USA. 2001;98(4):1583-1588.

49. Mudge JM, Harrow J. Creating reference gene annotation for the mouse C57BL6/J genome assembly. Mamm Genome. 
2015;26(9-10):366-378.

50. Dobin A, et al. STAR: ultrafast universal RNA-seq aligner. Bioinformatics. 2013;29(1):15-21.

51. Li H, et al. The Sequence Alignment/Map format and SAMtools. Bioinformatics. 2009;25(16):2078-2079.

52. Liao Y, Smyth GK, Shi W. featureCounts: an efficient general purpose program for assigning sequence reads to genomic features. Bioinformatics. 2014;30(7):923-930

53. Love MI, Huber W, Anders S. Moderated estimation of fold change and dispersion for RNA-seq data with DESeq2. Genome Biol. 2014;15(12):550 